\title{
Loneliness, Mindfulness, and Academic Achievements: A Moderation Effect among First-Year College Students
}

\author{
Eyal Rosenstreich* and Malka Margalit
}

Department of Behavioral Sciences, Peres Academic Center, Israel

\begin{abstract}
The transition to college enhances feelings of loneliness, and lonely students typically underperform academically due to the depletion of cognitive resources. Mindfulness practice has been demonstrated to improve certain cognitive abilities. The current study examined whether mindfulness practice may moderate the relations between perceived loneliness and academic performance. In this study we combined data from two separate studies. In the first, loneliness, optimism, and academic self-efficacy were measured at the beginning of the academic year. In the second, mindfulness workshops were conducted in order to examine their impact on memory performance. In all, 73 college students in their first semester participated in the conjoint study $(N=50$ mindfulness practice and $N=23$ control $)$. Their grades at the end of the semester and at the end of the year were also collected. Regression analyses were conducted in order to examine whether mindfulness moderated the effect of loneliness on academic achievements, and revealed that: a) loneliness was negatively correlated with recognition memory performance and with grades; b) grades were higher in the mindfulness group than in the control group; c) mindfulness and loneliness interacted, such that loneliness negatively predicted academic grades only for students who did not practice mindfulness. The results suggested that mindfulness practice may reduce the outcomes of loneliness on academic achievements in the first year of college.
\end{abstract}

Keywords: Academic achievements, Cognitive resources, Loneliness, Mindfulness.

Loneliness is one's subjective feeling of social isolation, regardless of one's actual social status [1]. Loneliness may affect people of different ages and social statuses, and may derive from different sources. Specifically, a feeling of loneliness may be induced by the absence or loss of a life partner or best friend (i.e. emotional loneliness), an inability to belong to a desired social group (i.e. social loneliness), experiences of alienation and isolation (i.e. existential loneliness), and by the perceived notion that one cannot be understood by others (i.e. representational loneliness [1]. The transition to college normatively involves major network changes, and it enhances the experience of loneliness [2].

Loneliness has harmful effects on physical, emotional, and cognitive wellbeing [3, 4]. In particular, feeling lonely has been demonstrated to be associated with increased blood pressure [5], with increased risk for cardiovascular diseases [6], with a decreased self-efficacy, and with an increase in depressive symptoms [7]. Most important to our study, loneliness has been demonstrated to predict cognitive decline among the elderly $[8,9]$ and was suggested to be associated with inferior cognitive and emotional control [10]. Specifically, as compared to those who are not lonely, lonely participants showed greater difficulty in regulating auditory attention, which manifested in an impaired ability to attenuate irrelevant information $[11,12]$.

*Address correspondence to this author at the Department of Behavioral Sciences, Peres Academic Center, 10 Peres St. Rd., Rehovot, Israel; Tel/Fax: +972-8-9378914; E-mail: eyal@pac.ac.il
In a comprehensive meta-analysis, Masi, et al. [13] examined the efficiency of different interventions to reduce loneliness and its effects. They concluded that interventions designed to improve social cognition [14] were found to be effective in decreasing loneliness. Such interventions promote the changing of maladaptive social cognition. Specifically, methods that promote redirecting one's attention from the search for social threats may reduce social-specific ruminations, induce more positive affect, and thus decrease the cognitive load induced by loneliness $[10$, $15]$.

In the current study we propose that employing interventions aimed at improving cognitive performance without tapping directly onto aspects of social cognition, may be revealed as an efficient approach for reducing the maladies of loneliness. Such interventions, namely meditation-based interventions, are low cost, highly efficient and easy to employ [16]. In the current study we focus on mindfulness meditation practice.

Mindfulness meditation, a growing field of interest in psychology, is a means of cultivating an awareness of the ongoing experience [17] and accepting the world as it is [18]. The practice of mindfulness mainly consists of directing attention to (or becoming aware of) breathing, everyday functions (e.g., the way you eat or walk), and sensory input (e.g., become aware of the feeling at the tip of your index finger). Along with the practice of attentiveness, mindfulness practice entails the cultivation of nonjudgmental perspective of the self and others [19]. Being 
non-judgmental enables one to let go of unpleasant thoughts or experiences, instead of ruminating about it.

Mindfulness is rather easy to practice, and although the canonical practice protocol consists of eight weeks of practice [17] the positive effects of mindfulness are evident even after a short practice [20]. For example, Mindfulness has been demonstrated to increase positive affect [21], to be positively correlated with the ability to tolerate distressful tasks [22], and to improve cognitive performance to some extent [16, 23, 24]. Moreover, mindfulness practice has been recently demonstrated to reduce the level of loneliness among the elderly [20].

Because loneliness is associated with increased stress and decreased cognitive resources [3], it seems that mindfulness and loneliness may have opposite effects on similar cognitive and emotional structures [25]. Therefore, we propose that mindfulness practice may reduce the impact of loneliness on behavior. We had the opportunity to examine this notion by crossing data from two studies conducted during the first semester of the 2013 academic year. The first study aimed to examine the relations between loneliness, hope, and academic self-efficacy and their effect on academic achievements among college students during their transition at the beginning of their first academic year. The second study was conducted about a month later and was aimed to examine the impact of mindfulness practice on memory. Specifically, students participated in mindfulness workshops in which they acquired the basics of mindfulness meditation practice, and they were compared with control participants in various memory measures [26]. We combined data from both studies along with the students' mean grades at the end of the first semester (i.e. immediately after the second study had been completed) and at the end of the academic year.

We hypothesized that loneliness would be related to lower academic achievements, while mindfulness practice would yield higher grades. Moreover, we hypothesized that mindfulness and loneliness would interact, such that mindfulness practice would moderate the effects of loneliness. Specifically, we predicted that loneliness would be associated with low grades only among students in the control group. For students in the mindfulness group, no effect of loneliness on academic performance will be found.

\section{METHOD}

\section{Participants}

The sample consisted of 73 first-year students from the behavioral sciences department at the Peres Academic Center, sampled in a non-probability sampling. They were divided into two groups: a mindfulness workshop group $(\mathrm{N}=50$ students: 47 females, mean age $=25.01, \mathrm{SD}=6.49)$ and a control group $(\mathrm{N}=23$ students: 22 females, mean age $=25.02, \mathrm{SD}=4.95)$. A Chi Square analysis indicated that there was an equal distribution of males and females in both groups, $\chi^{2}=0.083, p=.773$. Both groups were of the same cohort and were sampled from an Introduction to Statistics course, which took place in the first semester of 2013. Participants' mean age was common to the mean age of freshmen students in Israel (which is higher than in the US due to a 3-years mandatory military service).

\section{Design and Materials}

The study consisted of one between-subjects grouping variable (Group: mindfulness practice, control) and six measures: loneliness, academic self-efficacy, optimism, memory performance, mean grades at the end of the first semester, and mean grades at the end of the academic year.

\section{The Mindfulness Workshops}

The mindfulness workshops consisted of five 30-minute weekly sessions, during which mindfulness meditation exercises were introduced and practiced [19]. In the first session, breathing meditation and body scan were practiced. In sessions 2-5 various mindfulness exercises were practiced, such as an imaginary mindful walk in a forest, imaginary mindful chocolate eating, and concentrating on auditory and tactile stimuli. At the end of each session, participants were e-mailed with written and recorded instructions to practice at home, and were asked to maintain a daily routine of 15- to 30-minute practice. Two mindfulness workshops were conducted consecutively, from mid-semester to its end $(N=29$ in the first workshop and $N=21$ in the second workshop). Due to time constraints, the second workshop consisted of four sessions instead of five.

\section{Memory Performance}

Memory performance was assessed using a common task, the Deese-Roediger-McDermott paradigm (DRM; [27]). At the study stage, 100 words were presented on an A4 sheet of paper in 10 rows of 10 words each. At the test stage, 80 words were presented, of which 40 were studied (i.e. old) and 40 were unstudied (i.e. new) words. Test words were presented on an A4 sheet of paper, and participants determined for each word whether it had been presented at study or not. See Appendix for detailed description of the memory procedure.

\section{Loneliness}

The Hebrew adaptation [28] of the loneliness scale [29] consisted of nine statements describing social and emotional loneliness (e.g. "I miss having a really close friend."). The measure uses a 3-point Likert scale from 1 (no) to 3 (yes). A Cronbach alpha of 0.71 was obtained in previous studies, and in our study it was 0.82 .

\section{Academic Self-Efficacy (ASE)}

The Hebrew adaptation [30] of the self-efficacy for selfregulated learning scale [31] consists of 11 statements describing students' beliefs about how they can cope with various academic tasks in order to succeed in their studies and self-regulate their learning activities (e.g. "I can remember what has been studied in class and in the textbook."). The measure uses a 7-point Likert scale with endpoints of 1 (not sure at all) and 7 (completely confident). A Cronbach alpha of 0.87 was obtained for the measure in earlier studies. In the current study, the alpha was 0.91 . 


\section{Optimism}

Dispositional optimism was measured using the Hebrew adaptation of the Life Orientation Test (LOT-R; [32]). This scale contains 8 items rated on a 5 -point scale $(1=$ strongly agree, 5 = strongly disagree), with a high score indicating an optimistic tendency. The internal reliability and test-retest of the original version were satisfactory $(\alpha=0.76$, test-retest $=$ 0.79; Scheier \& Carver, 1985). In our study, the alpha was 0.66 .

\section{Academic Achievements}

Academic achievements were assessed as the mean grade at two time points: the end of the first semester and the end of the entire academic year. Because the mindfulness workshops were conducted in the middle of the first semester, the mean grade from the first semester reflects academic achievements one month after the workshops had ended. Thus, the mean grade of the entire year reflects academic achievements within eight months after the workshops had ended.

\section{Procedure}

In the present study we combined data collected from the same participants in two separate studies. The first study was conducted at the beginning of the academic year 2012-2013. This study was aimed at examining the relations between academic achievements and various measures of psychological wellbeing. In this study, participants filled the loneliness, optimism, and academic self-efficacy questionnaires. Upon completion of this study, participants received a five-point participation bonus to their grade in Introduction to Psychology. The second study was conducted at the middle of the semester (about one month after the data in the first study were collected). This study investigated the impact of mindfulness meditation on memory performance in general, and on the production of false memories in particular. Participants in the mindfulness workshops practiced mindfulness for four or five weekly sessions, and also instructed to practice at home. Memory performance was measured at the beginning and at the end of the workshops. We also administrated the memory assessment to a group of same-cohort, naïve participants, who participated in the first study but did not participated in the mindfulness

Table 1. Means, (SDs), and $\mathbf{N}$ of the wellbeing, cognitive, and academic measures as a function of Group.

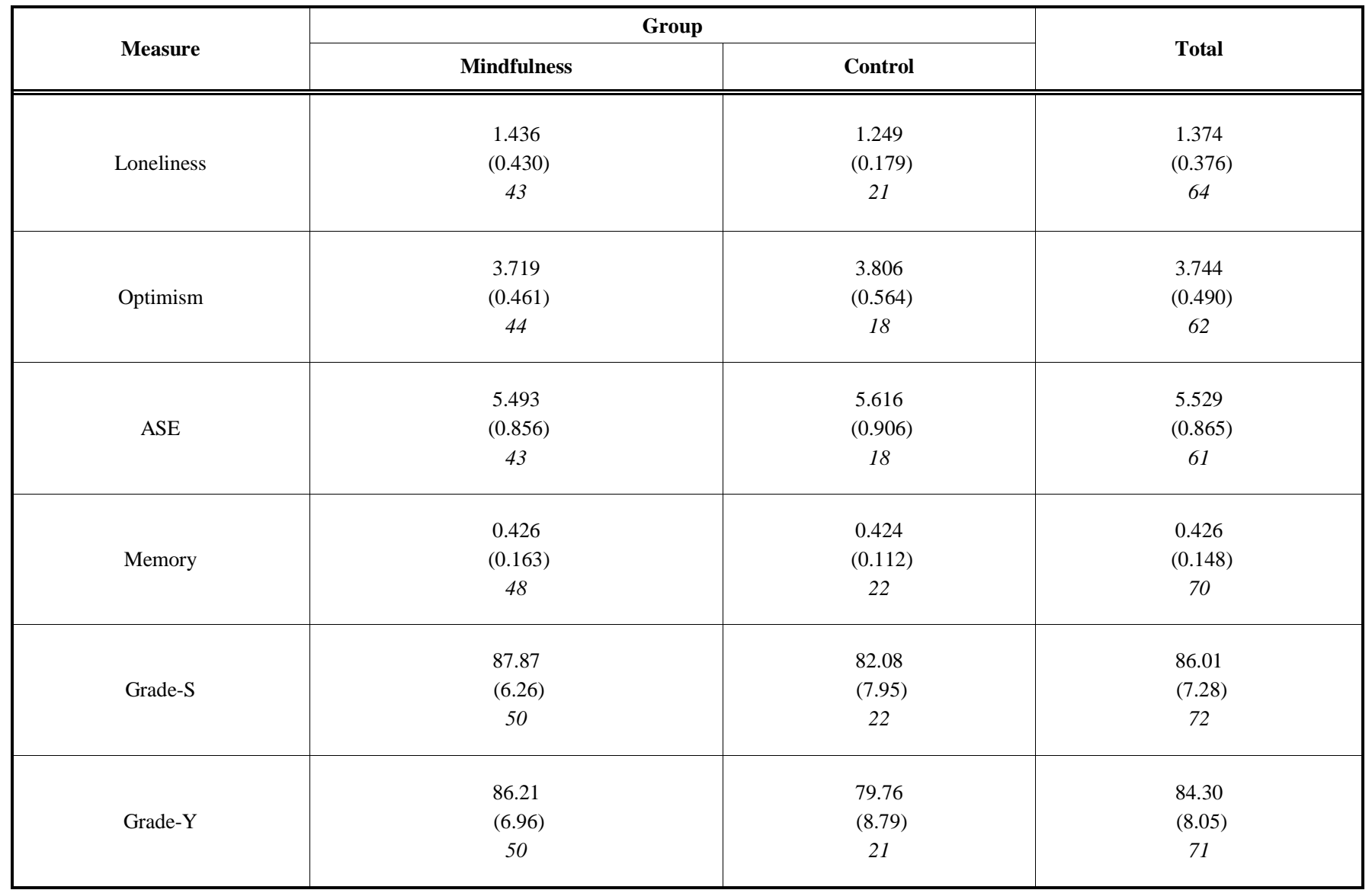

Note. ASE $=$ academic self-efficacy. Memory $=$ memory performance score. Grade-S and Grade- $Y=$ mean grade at the end of the first semester and at the end of the academic year, respectively. Standard deviations are presented in parentheses. The number of cases is presented in italics. 
workshops. Data was combined between studies based on participants' identification number. Participants were excluded from analysis if they were not affiliated with either the mindfulness or control groups, if their loneliness score was not recorded, or if their grades were unavailable.

\section{RESULTS}

\section{Preliminary Analyses}

For each participant, mean scores were calculated in the loneliness, optimism, and academic self-efficacy questionnaires, such that a high score indicated a high rate of loneliness, optimism, and academic self-efficacy, respectively. In addition, for each participant, the memory performance score were calculated as presented in the Appendix. The memory measure ranged from 0 to 1 , with 0 representing guessing. Memory performance was assessed in the mindfulness group using pre-intervention data, thus memory performance scores in the mindfulness and control groups represent initial, unmanipulated memory rates. Detailed descriptive statistics of all measures in the study are presented in Table 1.

\section{Loneliness and Cognition}

We first examined whether loneliness was related to cognitive functioning. To this end, multiple regression analysis was conducted, with memory performance scores as the predicted variable and with loneliness, optimism, and academic self-efficacy scores as the predictors. The predictors were entered simultaneously to the analysis. Table 2 presents the regression coefficients.

Table 2 reveals that loneliness negatively predicted to memory performance, such that high loneliness scores were associated with low memory performance. Optimism and academic self-efficacy did not predict memory performance. We next turned to examine the joint effects of loneliness and mindfulness on academic achievements.

\section{The Joint Effects of Loneliness and Mindfulness}

In order to examine the joint effects of loneliness and mindfulness on academic achievements, we first conducted a one-way ANOVA analysis, with Group (mindfulness, control) as the independent variable and Loneliness scores as the dependent variable (see Table $\mathbf{1}$ for means and SDs). The analysis revealed that the groups differed in their preintervention loneliness scores, such that participants in the mindfulness group felt lonelier than participants in the control group, $F(1,62)=3.636, p=0.061, \eta^{2}=0.055$.

Next, we examined our main hypothesis that Group and Loneliness would interact. Specifically, we examined whether mindfulness would increase academic achievements, as compared to control, but only for participants with high loneliness scores. To this end, we conducted a hierarchical regression with Group (mindfulness, control) and Loneliness as predictors and Grade- $\mathrm{Y}$ as the predicted variable. All variables were mean centered, and an interaction was computed and entered into the model (see 33 for details). Regression coefficients are presented in Table $\mathbf{3}$.

Table 3 reveals that Group positively predicted academic achievements, with the mindfulness group achieving higher average grades than the control participants. In addition, Loneliness negatively predicted academic achievements, such that high loneliness scores were associated with low academic performance. Most importantly, Group and Loneliness interacted significantly. Simple effects of the interaction were extracted using Hayes' PROCESS procedure [33] and are presented in Fig. (1).

Fig. (1) reveals that, as predicted, participants in the mindfulness and control groups did not differ in their academic achievements if they had low loneliness scores. In contrast, participants with medium or high loneliness scores achieved high grades at the end of the year if they participated in the mindfulness workshops, as compared to the control group. This interaction was observed also when Grade-S served as the predicted variable $(B=29.54, S E=$ $\left.9.99, \Delta R^{2}=.142, p=.005\right)$, as well as when Optimism and

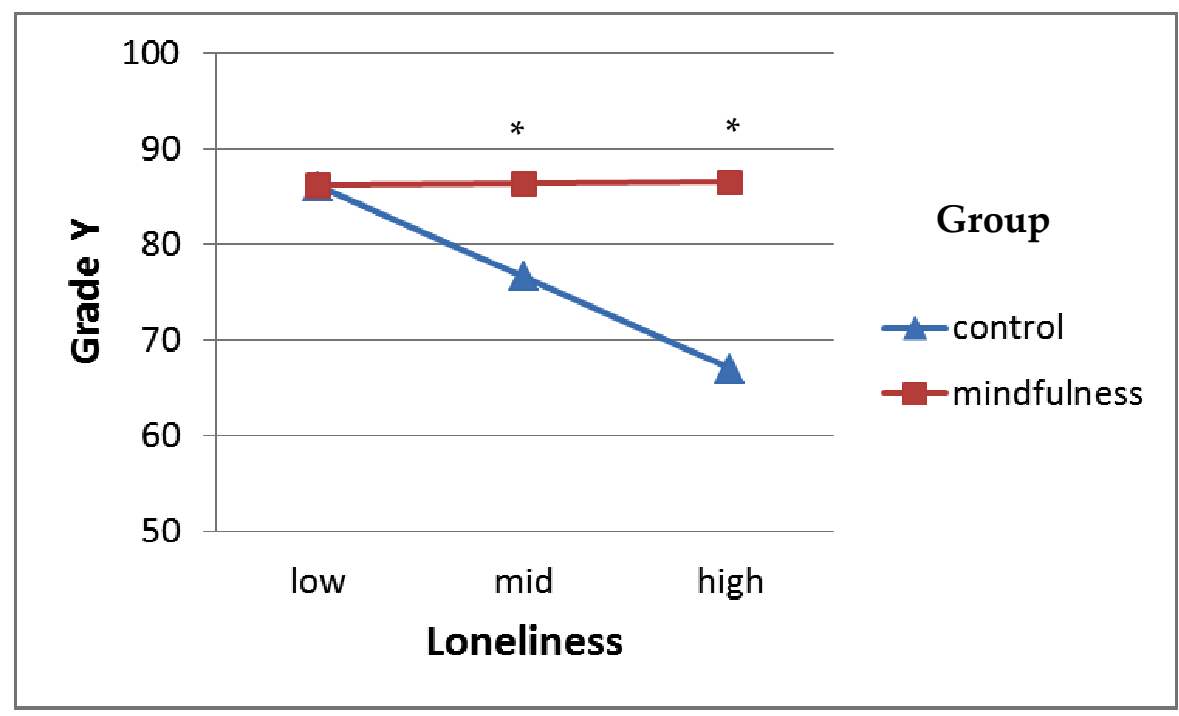

Fig. (1). Interaction effect between Group and Loneliness on Academic Achievements.

Note. Grade-Y = mean grade at the end of the first academic year. Low, Mid, and High levels of loneliness represent $z=-1, z=0$, and $z=1$, respectively, in the transformed-to-normal loneliness scores. $* p<.001$ for the comparison between the mindfulness and control groups. 
Table 2. Summary of regression analysis for variables predicting memory performance $(\mathrm{N}=51)$.

\begin{tabular}{|c|c|c|c|}
\hline Variable & $\boldsymbol{B}$ & $\boldsymbol{S E} \boldsymbol{B}$ & $\boldsymbol{\beta}$ \\
\hline \hline Loneliness & -0.142 & 0.061 & $-0.372^{*}$ \\
\hline Optimism & -0.006 & 0.049 & -0.020 \\
\hline ASE & -0.009 & 0.026 & -0.053 \\
\hline$R^{2}$ & & 0.122 & $2.215^{\mathrm{a}}$ \\
\hline$F$ for $R^{2}$ & & & \\
\hline
\end{tabular}

Note: ASE $=$ academic self-efficacy.

${ }^{\mathrm{a}} p=0.098, * p<0.05$

Table 3. Summary of Hierarchical Regression Analysis for Group and Loneliness as predictors of the mean grade at the end of the year $(\mathbf{N}=63)$.

\begin{tabular}{|c|c|c|c|c|c|c|}
\hline \multirow{2}{*}{ Variable } & \multicolumn{3}{|c|}{ Model 1} & \multicolumn{3}{|c|}{ Model 2} \\
\hline & $\boldsymbol{B}$ & $S E B$ & $\beta$ & $\boldsymbol{B}$ & $S E B$ & $\boldsymbol{\beta}$ \\
\hline Group & 6.888 & 2.067 & $0.406^{* *}$ & 9.742 & 2.218 & $0.570 * * *$ \\
\hline Loneliness & -1.496 & 2.562 & -0.071 & -7.613 & 3.291 & $-0.359 *$ \\
\hline Group x Loneliness & & & & 25.319 & 9.182 & $0.430 * *$ \\
\hline$R^{2}$ & \multicolumn{3}{|c|}{$\begin{array}{c}0.157 \\
5.568 * *\end{array}$} & \multicolumn{3}{|c|}{$\begin{array}{c}0.253 \\
7.603 * *\end{array}$} \\
\hline
\end{tabular}

ASE were held as covariates $\left(B=33.98, S E=11.06, \Delta R^{2}=\right.$ $.144, p=.004)$.

\section{DISCUSSION}

The aim of this study was to investigate whether mindfulness practice may moderate the effects of loneliness on academic achievements. In order to investigate this notion, we combined data collected in two studies in which levels of loneliness, academic self-efficacy, and optimism were collected at the beginning of the academic year, and mindfulness meditation workshops were conducted about a month later.

As hypothesized, loneliness negatively predicted academic achievements, such that students with high loneliness scores were more likely to achieve lower grades than students with low loneliness scores would. Furthermore, mindfulness predicted academic achievements, such that students who participated in the mindfulness workshops were more likely to achieve higher grades than students who did not participate in the workshops. These findings further support the notion that loneliness may impair the lonely person's performance [3, 10]. Also, it provides further support for the beneficial impact of meditation in generaland mindfulness in particular - on academic performance [34-36]. More importantly, as predicted, our findings showed an interaction between mindfulness and loneliness, such that mindfulness practice moderated the effects of loneliness on academic achievements. In fact, it seemed that mindfulness compensated for the effects of loneliness, even when academic self-efficacy and optimism were controlled.

We suggest three possible pathways that may serve as mechanisms through which mindfulness moderates the effects of loneliness on academic performance (see also 26). The first is the cognitive pathway, which refers to enhanced memory, attention, and executive function performance evoked by mindfulness practice [24, 37, 38]. Loneliness has been argued to be intimately related to cognitive deficiencies, such as difficulty in attention disengagement [39] and heightened memory attunement toward social information [40]. Hence, mindfulness may reduce the cognitive effects of loneliness by increasing memory [26, 41] and attention [42], or by reducing the role of emotions in the processes of perception and decision making [43].

The second pathway through which mindfulness may reduce the effects of loneliness is the interpretation of internal signals of stress. A subjective feeling of loneliness is typically characterized by increased rates of stress, anxiety, and cortisol levels [3]. These measures have been demonstrated to be reduced by mindfulness practice [42]. Thus, because academic performance is highly sensitive to emotional wellbeing [44-46], mindfulness may serve as a protective agent against the manifestations of decreased wellbeing in the academic setting.

Finally, the third pathway that we propose to explain how mindfulness may reduce the effects of loneliness on academic achievement, is initiating changes in selfperception. Loneliness typically evokes-especially in children and adolescents-low self-esteem [47], challenging their self-worth and giving them a feeling of incompetence [48]. This negative perception of the self may be manifested in low academic self-efficacy [1], and thus result in poor academic performance. Mindfulness promotes the cultivation of acceptance and self-compassion [49] and has been demonstrated to be positively correlated with academic selfefficacy [44]. Taken together, it seems mindfulness may decrease the effects of loneliness on academic performance by debiasing the lonely student's perceived competence.

Due to the design of our study, we were not able to conclude which of the three proposed pathways underlie the 
observed moderation effect of mindfulness on loneliness and academic achievements. Specifically, because the mindfulness study was aimed at investigating the impact of mindfulness on memory, data regarding students' stress, general self-efficacy, or attention-which are needed in order to understand the connection between mindfulness and academic achievements in the context of loneliness-were not collected. Furthermore, although our data showed that loneliness was negatively correlated with memory performance and that mindfulness increased memory performance, memory performance did not predict academic performance. Thus, further studies are needed in order to clarify the underlying paths between mindfulness, loneliness, and academic achievements.

An unexpected finding showed that students who participated in the mindfulness workshops reported experiencing higher levels of loneliness in comparison to control students. This finding entails two directions for future studies. First, this finding may indicate a tendency for help-seeking among lonely students. In the case of the mindfulness workshops, students were aware that these workshops were aimed at improving memory and attention. It is possible that the lonely students identified in these workshops an opportunity to break the cycle of loneliness. Second, this finding may enrich our knowledge of the characteristics of those who agree with and are able to commit to the demands of mindfulness practice.

\section{CONCLUSION, LIMITATIONS, AND FUTURE DIRECTIONS}

In summary, our study showed that mindfulness practice may moderate the effects of loneliness on academic achievements. This finding entails important implications regarding students' psychological wellbeing and academic performance. Specifically, it seems that difficulties in academic performance can be approached, to some extent, using mindfulness. Hence, mindfulness, as an easy-toemploy self-help intervention, might serve as a front line treatment for misadjusted students or for students with learning difficulties.

Nevertheless, these conclusions are constrained by two caveats. First, because the data analyzed in this study were collected in two independent studies, participants were not randomly allocated to the mindfulness and control groups. However, in our opinion, this caveat may have little effect, if any, on the findings obtained in this study, because all participants were sampled from a pool of same-cohort students. Furthermore, not being randomized, the allocation to the groups resulted in the mindfulness group having higher mean loneliness scores than the control group. Because loneliness negatively predicted the mean grade at the end of the year, such an a priori difference between the groups only strengthens our findings: The mindfulness group not only achieved higher grades than the control group, but they did so with higher loneliness scores to begin with. Indeed, it could be argued that the two groups differed in their academic skills to begin with. Although we cannot rule out this possibility, it should be mentioned that all our participants were students with low academic starting point; most of them without a high school diploma or a sufficient SECHEM score (The Israeli equivalent to the American
GPA) needed for university enrolment. Therefore, it is not likely that a priori differences in academic abilities confounded our findings.

The second caveat in our study is that loneliness scores were only collected before the mindfulness intervention. The absence of data regarding loneliness scores following the mindfulness intervention makes it impossible to conclude anything on the impact of mindfulness practice on loneliness. More importantly, we were not able to examine whether changes in loneliness scores mediated the connection between mindfulness and academic achievements, nor were we able to examine a three-way interaction between mindfulness, loneliness, and cognition and their effect on academic achievements.

Thus, future studies should examine the impact of mindfulness on loneliness and academic achievements in a randomized research design, with loneliness being measured after the mindfulness workshop has ended. Furthermore, a full factorial design may be employed by manipulating loneliness as well as mindfulness. That is, loneliness may be induced experimentally [50] within each of the mindfulness and control groups. Inducing loneliness may enable researchers to make conclusions about first and second order mediation-moderation connections between mindfulness, loneliness, cognition, and academic achievements.

\section{APPENDIX}

\section{A Detailed Description of the Memory Procedure Used in this Study}

\section{Memory Task}

In this study, memory performance was assessed using a common task, the Deese-Roediger-McDermott paradigm (DRM; 27). Although this task was primarily aimed at inducing false memories, it enables the extraction of an unmanipulated memory assessment. The DRM paradigm consisted of two stages, study and test. At the study stage, an A4 sheet of paper with 10 sets of 10 associatively related words (e.g. sugar, sour, cookie) was presented for two minutes. At the test stage, 40 studied (i.e. old) and 40 unstudied (i.e. new) words were presented on A4, and participants determined for each word whether it had been presented at study or not. See Appendix for detailed description of the memory procedure. The 40 old words were words which occupied the $2^{\text {nd }}, 4^{\text {th }}, 6^{\text {th }}$, and $8^{\text {th }}$ positions in each of the 10 sets of words presented at study. Similarly, the 40 new words occupied similar positions in 10 notpresented lists. To sum, participants' memory was assessed by a study list of 100 words, and a test list of 80 words (of which half were studied and half were not). The studied and unstudied words were randomly mixed and presented in four tables of 20 words each, in which one column contained the test words and two more columns were titled "studied" and "unstudied." For each word, participants were instructed to mark an " $X$ " in the column that best described their memory of the test words. Five random orders of the study and test lists were created. In addition, all DRM lists were counterbalanced, such that across participants, each DRM list appeared as both studied and unstudied. 


\section{Memory Scores}

First, the proportions of correct recognition of studied words (i.e. hit rate) and of false recognition of unstudied words (i.e. false-alarm rate) were calculated. The proportions were computed as the ratio between the number of words recognized and the total number of words with the same status (for rationale and further details, see [51]). That is, if a given participant correctly recognized 30 studied words out of a total of 40 studied words presented in the test, then his hit rate was:

$$
\mathrm{P}(\text { hit })=30 / 40=0.75
$$

Then, memory performance was derived from hit and false-alarm rates using a common method in memory research [52], in which false-alarm rates are subtracted from hit rates:

\section{Memory performance $=\mathrm{P}($ hit $)-\mathrm{P}($ false-alarm $)$}

Therefore, this measure of memory performance represents the extent to which correct memories exceeded memory intrusions. Hence, this measure ranges from 0 to 1 (values lower than 0 are theoretically possible, but not likely to occur), with 0 representing guessing. Memory performance was assessed in the mindfulness group using pre-intervention data, thus memory performance scores in the mindfulness and control groups represent initial, unmanipulated memory rates.

\section{CONFLICT OF INTEREST}

The authors confirm that this article content has no conflicts of interest.

\section{ACKNOWLEDGEMENTS}

Declared none.

\section{REFERENCES}

[1] Margalit M. Lonely children and adolescents: Self-perceptions, social exclusion, and hope. York: Springer 2010.

[2] Fiori KL, Consedine NS. Positive and negative social exchanges and mental health across the transition to college: Loneliness as a mediator. J Soc Pers Relat 2013: 30: 920-41.

[3] Hawkley LC, Cacioppo JT. Loneliness matters: a theoretical and empirical review of consequences and mechanisms. Ann Behav Med 2010; 40: 218-27.

[4] Cacioppo JT, Cacioppo S. Social relationships and health: the toxic effects of perceived social isolation. Soc Pers Psychol Compass 2014: 8: 58-72

[5] Hawkley LC, Thisted RA, Masi CM, Cacioppo JT. Loneliness predicts increased blood pressure: 5-year cross-lagged analyses in middle-aged and older adults. Psychol Aging 2010; 25: 132.

[6] Caspi A, Harrington H, Moffitt TE, Milne BJ, Poulton R. Socially isolated children 20 years later: risk of cardiovascular disease. Arch Pediatr Adolesc Med 2006; 160: 805.

[7] Wei M, Russell DW, Zakalik RA. Adult attachment, social selfefficacy, self-disclosure, loneliness, and subsequent depression for freshman college students: aa longitudinal study. J Couns Psychol 2005; 52: 602-14.

[8] Shankar A, Hamer M, McMunn A, Steptoe A. Social isolation and loneliness: relationships with cognitive function during 4 years of follow-up in the english longitudinal study of ageing. Psychosom Med 2013; 75: 161-70.

[9] Tilvis RS, Kähönen-Väre MH, Jolkkonen J, Valvanne J, Pitkala $\mathrm{KH}$, Strandberg TE. Predictors of cognitive decline and mortality of aged people over a 10-year period. J Gerontol A Biol Sci Med Sci 2004; 59: M268-M274.

[10] Cacioppo JT, Hawkley LC. Perceived social isolation and cognition. Trends Cogn Sci 2009; 13: 447-54.

[11] Cacioppo JT, Ernst JM, Burleson MH, et al. Lonely traits and concomitant physiological processes: the MacArthur social neuroscience studies. Int J Psychophysiol 2000; 35: 143-54.

[12] Baumeister RF, DeWall CN, Ciarocco NJ, Twenge JM. Social exclusion impairs self-regulation. J Pers Soc Psychol 2005; 88: 589-98.

[13] Masi CM, Chen HY, Hawkley LC, Cacioppo JT. A meta-analysis of interventions to reduce loneliness. Pers Soc Psychol Rev 2011; 15: 219-66.

[14] Williams A, Hagerty BM, Yousha SM, Horrocks J, Hoyle KS, Liu D. Psychosocial effects of the boot strap intervention in Navy recruits. Mil Med 2004; 169: 814-20.

[15] Baumeister RF, Twenge JM, Nuss CK. Effects of social exclusion on cognitive processes: anticipated aloneness reduces intelligent thought. J Pers Soc Psychol 2002: 83: 817-27.

[16] Gard T, Hölzel BK, Lazar SW. The potential effects of meditation on age-related cognitive decline: a systematic review. Ann N Y Acad Sci 2014: 1307: 89-103.

[17] Kabat-Zinn J. Mindfulness-based practices in context: Past, present, and future. Clin Psychol Sci Pract 2003; 10: 144-58.

[18] Phelan JP. Friendliness to the self. Mindfulness 2012; 3: 165-7.

[19] Shapiro SL, Carlson LE. The art and science of mindfulness: Integrating mindfulness into psychology and the helping professions. New York: APA publications 2009.

[20] Creswell JD, Irwin MR, Burklund LJ, et al. Mindfulness-based stress reduction training reduces loneliness and pro-inflammatory gene expression in older adults: a small randomized controlled trial. Brain Behav Immun 2012; 26: 1095-101.

[21] Brown KW, Ryan RM. The benefits of being present: mindfulness and its role in psychological well-being. J Pers Soc Psychol 2003; 84: 822-30.

[22] Feldman G, Dunn E, Stemke C, Bell K, Greeson J. Mindfulness and rumination as predictors of persistence with a distress tolerance task. Pers Indiv Differ 2014; 56: 154-8.

[23] Chiesa A, Calati R, Serretti A. Does mindfulness training improve cognitive abilities? A systematic review of neuropsychological findings. Clin Psychol Rev 2011; 31: 449-64.

[24] Rosenstreich E. Mindfulness and Memory. In: Singh NN, Ed Psychology of Meditation. New York: Nova 2014; pp. 167-85.

[25] Einav M, Rosenstreich E, Levi U, Margalit M. Coping with loneliness during childhood and adolescence. In: Rockach A, Sha'aked A, Eds. Treating loneliness. NY: Routledge in press.

[26] Rosenstreich E. Mindfulness and False-Memories: The Impact of Mindfulness Practice on the DRM Paradigm. J Psychol 2015; 1-17.

[27] Roediger HL III, McDermott KB. Creating false memories: Remembering words not presented in lists. J Exp Psychol Learn Mem Cogn 1995; 21: 803-14.

[28] Iecovich E. Psychometric properties of the hebrew version of the de jong gierveld loneliness scale. Educ Gerontol 2013; 39: 12-27.

[29] De Jong Gierveld J, Van Tilburg T. A 6-Item scale for overall, emotional, and social loneliness confirmatory tests on survey data. Res Aging 2006; 28: 582-98.

[30] Lackaye T, Margalit M. Self-efficacy, loneliness, effort, and hope: Developmental differences in the experiences of students with learning disabilities and their non-learning disabled peers at two age groups. Learn Disabil 2008; 6: 1-20.

[31] Zimmerman BJ. Self-Efficacy: An essential motive to learn. Contemp Educ Psychol 2000; 25: 82-91.

[32] Scheier MF, Carver CS. Optimism, coping, and health: Assessment and implication of generalized outcome expectancies. Health Psychol 1985; 4: 219-47.

[33] Hayes AF. PROCESS: A versatile computational tool for observed variable mediation, moderation, and conditional process modeling [White paper]. 2012: Retrieved from http://www.afhayes.com/ public/process2012.pdf

[34] Fiebert MS, Mead TM. Meditation and academic performance. Percept Motor Skill 1981; 53: 447-50.

[35] Hall PD. The effect of meditation on the academic performance of African American college students. J Black Stud 1999; 29: 408-15.

[36] Mrazek MD, Franklin MS, Phillips DT, Baird B, Schooler JW. Mindfulness training improves working memory capacity and GRE 
performance while reducing mind wandering. Psychol Sci 2013; 24: 776-81.

[37] Galla BM, Hale TS, Shrestha A, Loo SK, Smalley SL. The disciplined mind: Associations between the Kentucky Inventory of Mindfulness Skills and attention control. Mindfulness 2012; 3: 95103.

[38] Jha AP, Stanley EA, Kiyonaga A, Wong L, Gelfand L. Examining the protective effects of mindfulness training on working memory capacity and affective experience. Emotion 2010; 10: 54-64.

[39] DeWall CN, Maner JK, Rouby DA. Social exclusion and earlystage interpersonal perception: selective attention to signs of acceptance. J Pers Soc Psychol 2009; 96: 729.

[40] Gardner WL, Pickett CL, Jefferis V, Knowles M. On the outside looking in: loneliness and social monitoring. Pers Soc Psychol Bull 2005; 31: 1549-60.

[41] Lykins EL, Baer RA, Gottlob LR. Performance-based tests of attention and memory in long-term mindfulness meditators and demographically matched nonmeditators. Cog Therapy Res 2012; 36: $103-14$

[42] Tang Y, Ma Y, Wang J, et al. Short-term meditation training improves attention and self-regulation. PNAS 2007; 104: 17152-6.

[43] Hafenbrack AC, Kinias Z, Barsade SG. Debiasing the mind through meditation mindfulness and the sunk-cost bias. Psychol Sci 2014; $25: 369-76$
[44] Keye MD, Pidgeon AM. Investigation of the relationship between resilience, mindfulness, and academic self-efficacy. Open J Soc Sci 2013; $1: 1$.

[45] Napora L. Meditation in higher education: the question of change, a current problem, and evidence toward a solution. Biofeedback 2011; 39: 64-66.

[46] Davidson OB, Feldman DB, Margalit M. A focused intervention for 1st-year college students: promoting hope, sense of coherence, and self-efficacy. J Psychol 2012; 146: 333-52.

[47] Hall-Lande JA, Eisenberg ME, Christenson SL, Neumark-Sztainer D. Social isolation, psychological health, and protective factors in adolescence. Adolescence 2007; 42: 265-86.

[48] Qualter P, Munn P. The separateness of social and emotional loneliness in childhood. J Child Psychol Psychiatr 2002: 43: 233 44.

[49] Birnie K, Speca M, Carlson LE. Exploring self-compassion and empathy in the context of mindfulness-based stress reduction (MBSR). Stress Health 2010; 26: 359-71.

[50] Zhou X, Sedikides C, Wildschut T, Gao DG. Counteracting loneliness on the restorative function of nostalgia. Psychol Sci 2008; 19: 1023-29.

[51] Macmillan NA, Creelman CD. Signal detection theory: a user's guide (2nd ed.). Mahwah, NJ: Erlbaum 2005.

[52] Maddox GB, Naveh-Benjamin M, Old S, Kilb A. The role of attention in the associative binding of emotionally arousing words. Psychon Bull Rev 2012; 19: 1128-34.

(C) Rosenstreich and Margalit; Licensee Bentham Open.

This is an open access article licensed under the terms of the Creative Commons Attribution Non-Commercial License (http://creativecommons.org/licenses/by-nc/3.0/) which permits unrestricted, non-commercial use, distribution and reproduction in any medium, provided the work is properly cited. 